\title{
A LIFTING THEOREM AND UNIFORM ALGEBRAS
}

\author{
TAKAHIKO NAKAZI AND TAKANORI YAMAMOTO
}

\begin{abstract}
In this paper we discuss the possible generalizations of a lifting theorem of a $2 \times 2$ matrix to uniform algebras. These have applications to Hankel operators, weighted norm inequalities for conjugation operators and Toeplitz operators on uniform algebras. For example, the Helson-Szegö theorems for general uniform algebras follow.
\end{abstract}

1. Introduction. We will consider a fixed uniform algebra $A$ on a compact Hausdorff space $X$, and a fixed homomorphism $\tau$ in $M_{A}$, the maximal ideal space of $A$. The set of representing measures for $\tau$ will be denoted by $N_{\tau}$. The kernel of $\tau$ will be denoted by $A_{0}$. That is, $A_{0}$ consists of the functions $f$ in $A$ such that $\tau(f)=0$. We study only $2 \times 2$ measure matrices $\mu=\left(\mu_{i j}\right)$ whose elements are finite complex regular Borel measures, and satisfy the conditions:

$$
\mu_{11} \geqslant 0, \quad \mu_{22} \geqslant 0, \quad \mu_{12}=\bar{\mu}_{21} .
$$

For a measure matrix $\mu=\left(\mu_{i j}\right)$, let us denote

$$
\mu\left[f_{1}, f_{2}\right]=\sum_{i, j=1,2} \int_{X} f_{i} \bar{f}_{j} d \mu_{i j} .
$$

If $\mu$ satisfies $\mu\left[f_{1}, f_{2}\right] \geqslant 0$ for all $f_{1}$ in $A$ and $f_{2}$ in $\overline{A_{0}}$, then $\mu$ is said to be a positive matrix on $A \times \overline{A_{0}}$. On the other hand, if $\mu$ satisfies $\mu\left[f_{1}, f_{2}\right] \geqslant 0$ for all $f_{1}$ and $f_{2}$ in $C(X)$, the algebra of continuous complex-valued functions on $X$, then $\mu$ is said to be a positive matrix on $C(X) \times C(X)$. If two measure matrices $\mu=\left(\mu_{i j}\right)$ and $v=\left(\nu_{i j}\right)$ satisfy $\mu_{11}=\nu_{11}, \mu_{22}=\nu_{22}$ and $\mu_{12}-\nu_{12}$ annihilates $A_{0}$, then we write $\mu \sim v$. If $\mu \sim v$, then $\mu=v$ on $A \times \overline{A_{0}}$.

It is known (cf. [6, Chapter II, Corollary 7.5]) that every complex measure $\mu_{i j}$ on $X$ has a unique decomposition

$$
d \mu_{i j}=W_{i j} d m_{i j}+d \mu_{i j}^{s} \quad(i, j=1,2)
$$

where $m_{i j}$ is some representing measure for $\tau, W_{i j}$ is a function in $L^{1}\left(m_{i j}\right)$ and $\mu_{i j}^{s}$ is supported on a Borel set $E$ such that $n(E)=0$ for all $n$ in $N_{\tau}$. Then, there exists a common representing measure $m$ for $\tau$ such that $d \mu_{i j}=W_{i j} d m+d \mu_{i j}^{s}(i, j=$ 1,2). In fact, we can take $m=\left(m_{11}+m_{12}+m_{21}+m_{22}\right) / 4$. We will call this

Received by the editors October 20, 1986.

1980 Mathematics Subject Classification. Primary 46J15, 47B35, 30D55, 32A35; Secondary 42B30, 47A20.

Key n'ords and phrases. Uniform algebra, lifting theorem, weighted norm inequality, Hankel operator, Toeplitz operator.

This research was partially supported by Grant-in-Aid for Scientific Research, Ministry of Education. 
decomposition the Lebesgue decomposition of a measure matrix $\mu=\left(\mu_{i j}\right)$ with respect to $N_{\tau}$. For a measure $\sigma$ defined by a function $s$ in $L^{1}(m)$ such that $\sigma(E)=\int_{E} s d m$ for all Borel sets $E$, we identify the measure $\sigma$ and the function $s$.

Proposition 1. Suppose that $\mu=\left(\mu_{i j}\right)$ is a positive matrix on $A \times \overline{A_{0}}$ (resp. $C(X) \times C(X))$. Let $d \mu_{i j}=W_{i j} d m+d \mu_{i j}^{s}(i, j=1,2)$ be its Lebesgue decomposition with respect to $N_{\tau}$. Then $\mathbf{W}=\left(W_{i j}\right)$ is positive on $A \times \overline{A_{0}}$ (resp. $\left.C(X) \times C(X)\right)$ and $\mu^{s}=\left(\mu_{i j}^{s}\right)$ is positive on $C(X) \times C(X)$.

Proof. Suppose $\mu=\left(\mu_{i j}\right)$ is positive on $A \times \overline{A_{0}}$. Let $E$ be an $F_{\sigma}$-set such that $n(E)=0$ for all $n$ in $N_{\tau}$. It follows from Forelli's Lemma (cf. [6, Chapter II, Lemma 7.3]) that $\chi_{E}$ lies in the $L^{2}\left(\left|\mu_{i j}^{s}\right|\right)$-closure of $A_{0}$. Since each $\mu_{i j}$ is a regular measure, the measure matrix $\mu^{s}=\left(\mu_{i j}^{s}\right)$ is positive on $C(X) \times C(X)$. On the other hand, if $f$ lies in $A$ (resp. $\left.A_{0}\right)$, then $\left(1-\chi_{E}\right) f$ lies in the $L^{2}\left(\left|\mu_{i j}\right|\right)$-closure of $A$ (resp. $\left.A_{0}\right)$. Hence $\mathbf{W}=\left(W_{i j}\right)$ is positive on $A \times \overline{A_{0}}$. This completes the proof.

Given a positive matrix $\mu$ on $A \times \overline{A_{0}}$, does there exist a positive matrix $\nu$ on $C(X) \times C(X)$ such that $\mu \sim v$ ? Recently, Arocena, Cotlar and Sadosky (see [1, 2, 4]) have shown that this is true when $A$ is a disc algebra. But it does not seem to be true for general uniform algebras, even for uniform algebras on finitely connected regions. More recently, the first author $[12,13]$ has given a new approach to this kind of problem for uniform algebras and he has studied the norms of Hankel operators, Helson-Szegö type theorems, and the left invertibility of Toeplitz operators for uniform algebras. The second author [15] has given another proof of the lifting theorem for a disc algebra and he has studied Helson-Szegö type theorems and Koosis type theorems using the lifting theorem for the disc algebra. The essential part of this paper is to give the lifting theorem for uniform algebras which contains the above results as corollaries.

Let $\mathscr{L}$ be the class of all positive functions on $X$ which are both bounded and bounded away from zero. For each measure matrix $\mu=\left(\mu_{i j}\right)$ which satisfies condition (\#), we will consider the set of measure matrices:

$$
[\mu]=\left\{\lambda=\left(\lambda_{i j}\right) \mid \begin{array}{l}
\left(d \lambda_{i j}\right)=\left(V_{i j} d m\right)+\left(d \mu_{i j}^{s}\right), \\
\left(V_{i j}\right)=\left(\begin{array}{ll}
v W_{11} & W_{12} \\
W_{21} & v^{-1} W_{22}
\end{array}\right) \text { for some } v \text { in } \mathscr{L}
\end{array}\right\}
$$

A measure matrix $\mu$ is an element of the set $[\mu]$ of measure matrices. Suppose $\boldsymbol{\mu}=\left(\mu_{i j}\right)$ is positive on $A \times \overline{A_{0}}$. If there exists a positive measure matrix $\boldsymbol{v}=\left(\nu_{i j}\right)$ on $C(X) \times C(X)$ such that $\mu \sim v$, then all $\lambda$ in $[\mu]$ are positive on $A \times \overline{A_{0}}$. In $\S 2$, we will prove the converse to this result, that is, if $\mu$ is positive on $A \times \overline{A_{0}}$ and if all $\lambda$ in $[\mu]$ are positive on $A \times \overline{A_{0}}$, then there exists a positive matrix $v$ on $C(X) \times$ $C(X)$ such that $\mu \sim v$. In $\$ 3$, we will treat some constants which are useful in studying Hankel operators, the Helson-Szegö type theorems and the Koosis type theorems. In $\S 4$, we will study the lifting theorem in the case when $N_{\tau}$ is finite dimensional. In $\S 5$, we will use the lifting theorem to study the norms of the Hankel 
operators. In $\S 6$, we will give the Koosis type theorems for the special uniform algebras. In $\S 7$, we will give the Helson-Szegö type theorems for the special uniform algebras and, in §8, some examples and another kind of lifting theorem will be given.

For each representing measure for $\tau$ in $M_{A}$ and $1 \leqslant p<\infty$ the Hardy space $H^{p}=H^{p}(m)$ (resp. $\left.H_{0}^{p}=H_{0}(m)\right)$ is the norm closure of $A$ (resp. $\left.A_{0}\right)$ in $L^{p}=$ $L^{p}(m)$. The weak-* closure of $A$ (resp. $A_{0}$ ) in $L^{\infty}=L^{\infty}(m)$ is denoted by $H^{\infty}=H^{\infty}(m)$ (resp. $H_{0}^{\infty}=H_{0}^{\infty}(m)$ ). For $1 \leqslant p \leqslant \infty$, let $K^{p}=\left\{f \in L^{p} ; \int_{X} f g d m\right.$ $=0$ for all $g$ in $\left.A_{0}\right\}$, and $K_{\delta}^{p}=\left\{f \in L^{p} ; \int_{X} f g d m=0\right.$ for all $g$ in $\left.A\right\}$. Then, $H^{p} \subseteq K^{p}$ and $H_{0}^{p} \subseteq K_{0}^{p}$.

2. The lifting theorem under a strong condition. The following theorem gives the lifting theorem which is discussed in the Introduction and is true for a general uniform algebra. Let $\left(H^{\infty}\right)^{-1}$ be the class of all invertible functions in $H^{\infty}$. If $A$ is a disc algebra, then $\mathscr{L}=\left|\left(H^{\infty}\right)^{-1}\right|=\left\{|h| ; h\right.$ is in $\left.\left(H^{\infty}\right)^{-1}\right\}$ (cf. [7, Chapter II, Theorem 4.5]). Hence the following theorem is a generalization of Arocena, Cotlar and Sadosky's theorem [2, p. 102].

TheOREM 2. Let $A$ be a uniform algebra on $X$, and let $\tau$ be in $M_{A}$. Suppose a measure matrix $\mu=\left(\mu_{i j}\right)$ satisfies condition (\#). Let

$$
d \mu_{i j}=W_{i j} d m+d \mu_{i j}^{s} \quad(i, j=1,2)
$$

be its Lebesgue decomposition with respect to $N_{\tau}$. The following conditions are then equivalent.

(i) All $\lambda$ in $[\mu]$ are positive on $A \times \overline{A_{0}}$.

(ii) There exists a positive measure matrix $v=\left(\nu_{i j}\right)$ on $C(X) \times C(X)$ such that $\mu \sim v$ and $\left(\mu_{i j}^{s}\right)=\left(\nu_{i j}^{s}\right)$.

Proof. We shall show that (i) implies (ii). Let $g$ be a function in $A_{0}$. For all natural numbers $n$, put $E_{n}=\{x \in X ;|g(x)|<1 / n\}$. We define functions $v_{n}$ by the formula

$$
v_{n}= \begin{cases}1 / n & \text { on } E_{n}, \\ |g|\left(\frac{n W_{22}+W_{11}+1}{n W_{11}+W_{22}+1}\right)^{1 / 2} & \text { off } E_{n} .\end{cases}
$$

It follows that $(1 / n)^{2} \leqslant v_{n} \leqslant n\|g\|_{\infty}$ a.e., hence $v_{n}$ is in $\mathscr{L}$. Since

$$
v_{n} W_{11} \leqslant \chi_{E_{n}} W_{11}+\left(1-\chi_{E_{n}}\right)|g|\left(W_{11}+W_{22}+1\right) \text { a.e. }
$$

and

$$
|g|^{2} v_{n}^{-1} W_{22} \leqslant \chi_{E_{n}} W_{22}+\left(1-\chi_{E_{n}}\right)|g|\left(W_{11}+W_{22}+1\right) \text { a.e., }
$$

it follows from Lebesgue's theorem that

$$
\begin{aligned}
\lim _{n \rightarrow \infty} \int_{X} v_{n} W_{11} d m & =\int_{X}|g|\left(W_{11} W_{22}\right)^{1 / 2}\left(1-\lim _{n \rightarrow \infty} \chi_{E_{n}}\right) d m \\
& \leqslant \int_{X}|g|\left(W_{11} W_{22}\right)^{1 / 2} d m
\end{aligned}
$$


and it follows in the same way that

$$
\lim _{n \rightarrow \infty} \int_{X}|g|^{2} v_{n}^{-1} W_{22} d m \leqslant \int_{X}|g|\left(W_{11} W_{22}\right)^{1 / 2} d m .
$$

Suppose

$$
\left(\begin{array}{cc}
v W_{11} & W_{12} \\
W_{21} & v^{-1} W_{22}
\end{array}\right)
$$

is positive on $A \times \overline{A_{0}}$ for all $v$ in $\mathscr{L}$. Then

$$
-2 \operatorname{Re} \int_{X} f_{1} \bar{f}_{2} W_{12} d m \leqslant \int_{X}\left|f_{1}\right|^{2} v_{n} W_{11} d m+\int_{X}\left|f_{2}\right|^{2} v_{n}^{-1} W_{22} d m
$$

for all $f_{1}$ in $A$ and $f_{2}$ in $\overline{A_{0}}$, and for all $n$. Put $f_{1}=1$ and $f_{2}=\bar{g}$. Let $n \rightarrow \infty$, then

$$
-\operatorname{Re} \int_{X} g W_{12} d m \leqslant \int_{X}|g|\left(W_{11} W_{22}\right)^{1 / 2} d m .
$$

Since this holds for all $g$ in $A_{0}$, it follows that

$$
\left|\int_{X} g W_{12} d m\right| \leqslant \int_{X}|g|\left(W_{11} W_{22}\right)^{1 / 2} d m .
$$

By the Hahn-Banach theorem, there exists a linear functional $T$ on $C(X)$ such that

$$
|T(G)| \leqslant \int_{X}|G|\left(W_{11} W_{22}\right)^{1 / 2} d m
$$

for all $G$ in $C(X)$, and

$$
T(g)=\int_{X} g W_{12} d m
$$

for all $g$ in $A_{0}$. Since $L^{1}\left(\left(W_{11} W_{22}\right)^{1 / 2}+1 / n\right)^{*}=L^{\infty}$ for all natural numbers $n$, there exists a function $s_{n}$ in $L^{\infty},\left\|s_{n}\right\|_{\infty} \leqslant 1$, such that

$$
T(G)=\int_{X} G s_{n}\left(\left(W_{11} W_{22}\right)^{1 / 2}+1 / n\right) d m .
$$

Taking a subsequence if necessary, $s_{n}$ then converges to some $s$ in $L^{\infty},\|s\|_{\infty} \leqslant 1$, in the weak-* topology. Put

$$
\mathbf{U}=\left(U_{i j}\right)=\left(\begin{array}{cc}
W_{11} & s\left(W_{11} W_{22}\right)^{1 / 2} \\
\bar{s}\left(W_{11} W_{22}\right)^{1 / 2} & W_{22}
\end{array}\right)
$$

Then $\mathbf{U} \sim \mathbf{W}$, since

$$
\int_{X} g U_{12} d m=\int_{X} g s\left(W_{11} W_{22}\right)^{1 / 2} d m=\int_{X} g W_{12} d m
$$

On the other hand,

$$
\begin{aligned}
-2 \operatorname{Re} \int_{X} F_{1} \bar{F}_{2} U_{12} d m & \leqslant 2 \int_{X}\left|F_{1} F_{2}\right|\left(W_{11} W_{22}\right)^{1 / 2} d m \\
& \leqslant \int_{X}\left|F_{1}\right|^{2} U_{11} d m+\int_{X}\left|F_{2}\right|^{2} U_{22} d m .
\end{aligned}
$$


Therefore,

$$
\sum_{i, j=1,2} \int_{X} F_{i} \bar{F}_{j} U_{i j} d m \geqslant 0
$$

for all $F_{1}, F_{2}$ in $C(X)$, and hence $\mathbf{U}=\left(U_{i j}\right)$ is positive on $C(X) \times C(X)$. Put $v=\left(\nu_{i j}\right), d \nu_{i j}=U_{i j} d m+d \mu_{i j}^{s}(i, j=1,2)$; then (ii) follows.

We shall show next that (ii) implies (i). Suppose $\lambda=\left(\lambda_{i j}\right)$ is an element of $[\mu]$. Then $d \lambda_{i j}=V_{i j} d m+d \mu_{i j}^{s}$, where

$$
\left(V_{i j}\right)=\left(\begin{array}{cc}
v W_{11} & W_{12} \\
W_{21} & v^{-1} W_{22}
\end{array}\right) .
$$

Since $\mu \sim \nu$, there exist integrable functions $U_{i j}$ such that $d \nu_{i j}=U_{i j} d m+d \mu_{i j}^{s}$. Furthermore, $W_{11}=U_{11}, W_{22}=U_{22}$ and

$$
\int_{X} f_{1} \bar{f}_{2} W_{12} d m=\int_{X} f_{1} \bar{f}_{2} U_{12} d m
$$

for all $f_{1}$ in $A$ and all $f_{2}$ in $\overline{A_{0}}$. Since $v$ is positive on $C(X) \times C(X)$, it follows from Proposition 1 that $\mathbf{U}=\left(U_{i j}\right)$ is positive on $C(X) \times C(X)$. Hence, $\left|U_{12}\right|^{2} \leqslant$ $U_{11} U_{22}$ a.e. Then

$$
\begin{aligned}
-2 \operatorname{Re} \int_{X} f_{1} \bar{f}_{2} V_{12} d m & \leqslant 2\left|\int_{X} f_{1} \bar{f}_{2} U_{12} d m\right| \\
& \leqslant 2 \int_{X}\left|f_{1} f_{2}\right|\left(U_{11} U_{22}\right)^{1 / 2} d m=2 \int_{X}\left|f_{1} f_{2}\right|\left(V_{11} V_{22}\right)^{1 / 2} d m \\
& \leqslant \int_{X}\left|f_{1}\right|^{2} V_{11} d m+\int_{X}\left|f_{2}\right|^{2} V_{22} d m .
\end{aligned}
$$

Therefore,

$$
\sum_{i, j=1,2} \int_{X} f_{i} \bar{f}_{j} V_{i j} d m \geqslant 0,
$$

which implies (i). This completes the proof.

Corollary 2.1. Suppose a matrix $\mathbf{W}=\left(W_{i j}\right)$ satisfies condition (\#). The following conditions are then equivalent.

(i)

$$
\left(\begin{array}{cc}
v W_{11} & W_{12} \\
W_{21} & v^{-1} W_{22}
\end{array}\right)
$$

is positive on $A \times \overline{A_{0}}$ for all $v$ in $\mathscr{L}$.

(ii) There exists a $k$ in $K^{1}$ such that

$$
\left|W_{12}-k\right|^{2} \leqslant W_{11} W_{22} \text { a.e. }
$$

Proof. We shall show that (i) implies (ii). It follows from (i) and Theorem 2 that there exists a positive matrix $v=\left(\nu_{i j}\right)$ on $C(X) \times C(X)$ such that $\left(W_{i j}\right) \sim\left(\nu_{i j}\right)$. Let $d \nu_{i j}=U_{i j} d m+d \nu_{i j}^{s}$ be the Lebesgue decomposition with respect to $N_{\tau}$, then 
$d \nu_{i j}^{s}=0$. Since $K^{1}$ annihilates $A_{0}$, there exists a $k$ in $K^{1}$ such that

$$
\boldsymbol{v}=\left(\begin{array}{cc}
W_{11} & W_{12}-k \\
W_{21}-\bar{k} & W_{22}
\end{array}\right) .
$$

Since $v$ is positive on $C(X) \times C(X)$, it follows that $\left|U_{12}\right|^{2} \leqslant U_{11} U_{22}$ a.e. This implies (ii). We shall show next that (ii) implies (i). Put

$$
v=\left(\begin{array}{cc}
W_{11} & W_{12}-k \\
W_{21}-\bar{k} & W_{22}
\end{array}\right) ;
$$

then this $v$ satisfies (ii) of Theorem 2, since $k$ annihilates $A_{0}$. This completes the proof.

Proposition 3. Suppose a matrix $\mathbf{W}=\left(W_{i j}\right)$ satisfies condition (\#). If $\mathbf{W}=\left(W_{i j}\right)$ is positive on $A \times \overline{A_{0}}$, then

$$
\left(\begin{array}{cc}
|h|^{2} W_{11} & W_{12} \\
W_{21} & |h|^{-2} W_{22}
\end{array}\right)
$$

is positive on $A \times \overline{A_{0}}$ for all $h$ in $\left(H^{\infty}\right)^{-1}$.

Proof. Let $h$ be in $\left(H^{\infty}\right)^{-1}, f_{1}$ be in $A$ and $f_{2}$ be in $\overline{A_{0}}$. Put $F_{1}=h f_{1}$ and $F_{2}=f_{2} / \bar{h}$. Then $F_{1}$ is in $H^{\infty}$ and $F_{2}$ is in $\bar{H}_{0}^{\infty}$. Since $H^{\infty}$ (resp. $H_{0}^{\infty}$ ) is a weak-* closure of $A$ (resp. $A_{0}$ ), by the hypothesis, we have

$$
\int_{X}\left|F_{1}\right|^{2} W_{11} d m+\int_{X}\left|F_{2}\right|^{2} W_{22} d m+2 \operatorname{Re} \int_{X} F_{1} \bar{F}_{2} W_{12} d m \geqslant 0 .
$$

Then

$$
\int_{X}\left|f_{1}\right|^{2}|h|^{2} W_{11} d m+\int_{X}\left|f_{2}\right|^{2}|h|^{-2} W_{22} d m+2 \operatorname{Re} \int_{X} f_{1} \bar{f}_{2} W_{12} d m \geqslant 0 .
$$

This completes the proof.

Arocena, Cotlar and Sadosky's theorem. Let $A$ be a disc algebra. Suppose a matrix $\mu=\left(\mu_{i j}\right)$ satisfies condition (\#). Let $d \mu_{i j}=W_{i j} d m+d \mu_{i j}^{s}(i, j=1,2)$ be its Lebesgue decomposition with respect to $N_{\tau}$. The following conditions are then equivalent.

(i) $\mu=\left(\mu_{i j}\right)$ is positive on $A \times \overline{A_{0}}$.

(ii) There exists a positive matrix $v=\left(\nu_{i j}\right)$ on $C(X) \times C(X)$ such that $\mu \sim v$.

(iii) $\left(\mu_{i j}^{s}\right)=\left(\nu_{i j}^{s}\right)$ and there exists $a k$ in $H^{1}$ such that

$$
\left|W_{12}-k\right|^{2} \leqslant W_{11} W_{22} \text { a.e. }
$$

Proof. We shall show that (i) implies (ii). If $\mu=\left(\mu_{i j}\right)$ is positive on $A \times \overline{A_{0}}$, then it follows from Proposition 1 that $\mu^{s}=\left(\mu_{i j}^{s}\right)$ is positive on $C(X) \times C(X)$ and $\mathbf{W}=\left(W_{i j}\right)$ is positive on $A \times \overline{A_{0}}$. By Proposition 3,

$$
\left(\begin{array}{cc}
|h|^{2} W_{11} & W_{12} \\
W_{21} & |h|^{-2} W_{22}
\end{array}\right)
$$


is positive on $A \times \overline{A_{0}}$ for all $h$ in $\left(H^{\infty}\right)^{-1}$. Since $A$ is a disc algebra, every $v$ in $\mathscr{L}$ can be written in the form $v=|h|^{2}$ a.e. for some $h$ in $\left(H^{\infty}\right)^{-1}$ (cf. [6, Chapter II, Theorem 4.5]). Hence

$$
\left(\begin{array}{cc}
v W_{11} & W_{12} \\
W_{21} & v^{-1} W_{22}
\end{array}\right)
$$

is positive on $A \times \overline{A_{0}}$ for all $v$ in $\mathscr{L}$. Therefore, all $\lambda$ in $[\mu]$ are positive on $A \times \overline{A_{0}}$. By Theorem 2, (ii) holds. We shall show that (ii) implies (iii). Since $\mu \sim \nu$, there exists integrable functions $U_{i j}$ such that $d \nu_{i j}=U_{i j} d m+d \mu_{i j}^{s}$. Furthermore, $W_{11}=$ $U_{11}, W_{22}=U_{22}$, and $W_{12}-U_{12}$ annihilates $A_{0}$. Then there exists a $k$ in $K^{1}$ such that $U_{12}=W_{12}-k$ a.e. Since $v$ is positive on $C(X) \times C(X)$, it follows from Proposition 1 that $\mathbf{U}=\left(U_{i j}\right)$ is positive on $C(X) \times C(X)$. Hence, $\left|U_{12}\right|^{2} \leqslant U_{11} U_{22}$ a.e. Therefore, $\left|W_{12}-k\right|^{2} \leqslant W_{11} W_{22}$ a.e. This implies (ii). Since $K^{1}$ annihilates $A_{0}$, (iii) implies (i). This completes the proof.

3. Constants relating to measure matrices. The following investigations will be useful in calculating the norms of Hankel operators and in studying the boundedness of some kinds of Riesz projections. We shall study only the absolutely continuous measure matrices which satisfy condition (\#).

The coset of an $f$ in $\left(L^{\infty}\right)^{-1}$ in $\left(L^{\infty}\right)^{-1} /\left(H^{\infty}\right)^{-1}$ is denoted by $(f)$. That is, $(f)=\left\{f h ; h\right.$ is in $\left.\left(H^{\infty}\right)^{-1}\right\}$. Define

$$
\begin{aligned}
\|(f)\| & =\inf \left\{\|g\|_{\infty}\left\|g^{-1}\right\|_{\infty} ; g \text { is in }(f)\right\}, \\
\gamma_{0} & =\sup \left\{\|(f)\| ; f \text { is in }\left(L^{\infty}\right)^{-1}\right\} .
\end{aligned}
$$

The first author [13] has used the constant $\gamma_{0}$ to show that the norms of Hankel operators are equivalent to the dual norms of $H^{1} \cdot \gamma_{0}$ may be finite or infinite. When $A$ is a disc algebra, $\gamma_{0}=1$.

Proposition 4. If $\mathbf{W}=\left(W_{i j}\right)$ is positive on $A \times \overline{A_{0}}$, then for each $v$ in $\mathscr{L}$,

$$
\left(\begin{array}{cc}
c v^{2} W_{11} & W_{12} \\
W_{21} & c v^{-2} W_{22}
\end{array}\right)
$$

is positive on $A \times \overline{A_{0}}$, where $0 \leqslant c \leqslant\|(v)\| \leqslant \gamma_{0}$.

Proof. Let $\varepsilon$ be any positive constant. Then there exists an $h$ in $\left(H^{\infty}\right)^{-1}$ such that $\|v h\|_{\infty}\left\|v^{-1} h^{-1}\right\|_{\infty} \leqslant\|(v)\|+\varepsilon$. Since the matrix $\mathbf{W}=\left(W_{i j}\right)$ is positive on $A \times \overline{A_{0}}$,

$$
\begin{aligned}
& -2 \operatorname{Re} \int_{X} f_{1} \bar{f}_{2} W_{12} d m \leqslant 2\left\{\int_{X}\left|f_{1}\right|^{2} W_{11} d m\right\}^{1 / 2}\left\{\int_{X}\left|f_{2}\right|^{2} W_{22} d m\right\}^{1 / 2} \\
& \leqslant 2\|v h\|_{\infty}\left\|v^{-1} h^{-1}\right\|_{\infty}\left\{\int_{X}\left|f_{1}\right|^{2} v^{2}|h|^{2} W_{11} d m\right\}^{1 / 2}\left\{\int_{X}\left|f_{2}\right|^{2} v^{-2}|h|^{-2} W_{22} d m\right\}^{1 / 2} \\
& \quad \leqslant(\|(v)\|+\varepsilon)\left\{\int_{X}\left|f_{1}\right|^{2} v^{2}|h|^{2} W_{11} d m+\int_{X}\left|f_{2}\right|^{2} v^{-2}|h|^{-2} W_{22} d m\right\}
\end{aligned}
$$


for all $f_{1}$ in $A, f_{2}$ in $\overline{A_{0}}$ and all $h$ in $\left(H^{\infty}\right)^{-1}$. Hence, the matrix

$$
\left(\begin{array}{cc}
(\|(v)\|+\varepsilon) v^{2}|h|^{2} W_{11} & W_{12} \\
W_{21} & (\|(v)\|+\varepsilon) v^{-2}|h|^{-2} W_{22}
\end{array}\right)
$$

is positive on $A \times \overline{A_{0}}$. By Proposition 3,

$$
\left(\begin{array}{cc}
(\|(v)\|+\varepsilon) v^{2} W_{11} & W_{12} \\
W_{21} & (\|(v)\|+\varepsilon) v^{-2} W_{22}
\end{array}\right)
$$

is positive on $A \times \overline{A_{0}}$. Let $\varepsilon \rightarrow 0$, then

$$
\left(\begin{array}{cc}
\|(v)\| v^{2} W_{11} & W_{12} \\
W_{21} & \|(v)\| v^{-2} W_{22}
\end{array}\right)
$$

is positive on $A \times \overline{A_{0}}$. This completes the proof.

If the matrix $\mathbf{W}=\left(W_{i j}\right)$ is positive on $A \times \overline{A_{0}}$, then it follows from Proposition 4 that the set of the constants

$$
\left\{c \geqslant 0 \mid\left(\begin{array}{cc}
c v^{2} W_{11} & W_{12} \\
W_{21} & c v^{-2} W_{22}
\end{array}\right) \text { is positive on } A \times \overline{A_{0}}\right\} .
$$

is not empty for each $v$ in $\mathscr{L}$. The infimum of the above set is denoted by $c(v, W)$. It follows from Proposition 4 that $c(v, W) \leqslant\|(v)\| \leqslant \gamma_{0}$.

Proposition 5. If $\mathbf{W}=\left(W_{i j}\right)$ is positive on $A \times \overline{A_{0}}$ and $v$ is in $\mathscr{L}$, then $c(v, W)=c(v|h|, W)$ for all $h$ in $\left(H^{\infty}\right)^{-1}$.

Proof. By Proposition 3, the above set which is defined for $v$ is the same as the set for $v|h|$. This completes the proof.

TheOREM 6. Suppose $\mathbf{W}=\left(W_{i j}\right)$ is positive on $A \times \overline{A_{0}}$. For each nonnegative constant $c$, the following conditions are equivalent.

(i) $\sup \{c(v, W) ; v$ is in $\mathscr{L}\} \leqslant c$.

(ii)

$$
\left(\begin{array}{cc}
c v^{2} W_{11} & W_{12} \\
W_{21} & c v^{-2} W_{22}
\end{array}\right)
$$

is positiver on $A \times \overline{A_{0}}$ for all $v$ in $\mathscr{L}$.

(iii) There exists a positive matrix $\mathbf{U}=\left(U_{i j}\right)$ on $C(X) \times C(X)$ such that

$$
\left(\begin{array}{cc}
c W_{11} & W_{12} \\
W_{21} & c W_{22}
\end{array}\right) \sim \mathbf{U}
$$

(iv) There exists a $k$ in $K^{1}$ such that

$$
\left|W_{12}-k\right|^{2} \leqslant c^{2} W_{11} W_{22} \text { a.e. }
$$

Proof. By the definition, (i) is equivalent to (ii). By Theorem 2, (ii) is equivalent to (iii). On the other hand, by Corollary 2.1, (ii) is equivalent to (iv). This completes the proof. 
THEOREM 7. Suppose $A$ is a uniform algebra such that $\gamma_{0}$ is finite. If a matrix $\mathbf{W}=\left(W_{i j}\right)$ is positive on $A \times \bar{A}_{0}$, then the following conditions are true.

(1) There exists a positive matrix $\mathbf{U}=\left(U_{i j}\right)$ on $C(X) \times C(X)$ such that

$$
\left(\begin{array}{cc}
\gamma_{0} W_{11} & W_{12} \\
W_{21} & \gamma_{0} W_{22}
\end{array}\right) \sim \mathbf{U}
$$

(2) There exists a $k$ in $K^{1}$ such that

$$
\left|W_{12}-k\right|^{2} \leqslant \gamma_{0}^{2} W_{11} W_{22} \text { a.e. }
$$

Proof. Since $c(v, W) \leqslant\|(v)\| \leqslant \gamma_{0}$, this theorem follows immediately from Theorem 6.

4. The finite-dimensional case. We assume in this section that the set $N_{\tau}$ of representing measures for $\tau$ is finite dimensional. Core measures always exist in this case. In fact, $N_{\tau}$ is the closure of its set of core points (cf. [6, Chapter IV, §5]). Let $m$ be a core point of $N_{\tau}$, and let $N^{\infty}=\left\{f \in L_{R}^{\infty} ; \int_{X} f g d m=0\right.$ for all $g$ in $\left.A\right\}$. Let $N_{c}^{\infty}$ be the complexification of $N^{\infty}$. Then $A+\overline{A_{0}}+N_{c}^{\infty}$ is weak-* dense in $L^{\infty}$ (cf. [6, Chapter IV, Theorem 6.2]).

Set $\mathscr{E}=\exp N^{\infty}=\left\{e^{u} ; u\right.$ is in $\left.N^{\infty}\right\}$. Then $\mathscr{E}$ is a subgroup of $\mathscr{L}$. $\mathscr{E}$ is defined by the first author [12].

THEOREM 8. Suppose $N_{\tau}$ is finite dimensional. Let $m$ be a representing measure for $\tau$. Suppose $\mathbf{W}=\left(W_{i j}\right)$ satisfies condition (\#). If

$$
\left(\begin{array}{cc}
v^{2} W_{11} & W_{12} \\
W_{21} & v^{-2} W_{22}
\end{array}\right)
$$

is positive on $A \times \overline{A_{0}}$ for all $v$ in $\mathscr{E}$, then it is positive on $A \times \overline{A_{0}}$ for all $v$ in $\mathscr{L}$. In this case,

$$
\sup \{c(v, W) ; v \text { is in } \mathscr{L}\}=\sup \{c(v, W) ; v \text { is in } \mathscr{E}\} \text {. }
$$

Proof. If $v$ is in $\mathscr{L}$, then there exists a $u_{0}$ in $N^{\infty}$ and a $u$ in the weak-* closure of $\operatorname{Re} A$ such that $\log v=u+u_{0}$ a.e. (cf. [6, Chapter IV, Theorem 6.2]). It follows that $u=\log |h|$ for some $h$ in $\left(H^{\infty}\right)^{-1}$. Put $v_{0}=e^{u_{0}}$; then $v_{0}$ is in $\mathscr{E}$. It follows from Proposition 3 that the above matrix is positive on $A \times \overline{A_{0}}$ for $v=|h| v_{0}$. Then, it follows from Proposition 5 that $c(v, W)=c\left(v_{0}, W\right)$. This completes the proof.

Theorem 8 assures us that the equivalence in Theorem 2 or Corollary 2.1 is also true for "all $v$ in $\mathscr{E}$ " instead of "all $v$ in $\mathscr{L}$." We should mention that in this case $K^{1}=H^{1}+N_{c}^{\infty}$ (cf. [6, Chapter IV, Theorem 6.2]). If $\operatorname{dim} N_{\tau}=0$, then $\mathscr{E}=\{1\}$. We assume now that $m$ is a unique logmodular measure. Then the linear span of $N^{\infty} \cap \log \left|\left(H^{\infty}\right)^{-1}\right|$ is equal to $N^{\infty}$ (cf. [6, Chapter IV, Corollary 7.6]).

THEOREM 9. Suppose $m$ is a unique logmodular measure for $\tau$, and a matrix $\mathbf{W}=\left(W_{i j}\right)$ is positive on $A \times \overline{A_{0}}$. Then $\sup \{c(v, W) ; v$ is in $\mathscr{L}\}$ is finite. The supremum is attained with some $v$ in $\mathscr{E}$.

Proof. It follows from Theorem 8 that the supremum in this theorem is equal to the supremum for all $v$ in $\mathscr{E}$. Choose $h_{1}, \ldots, h_{n}$ in $\left(H^{\infty}\right)^{-1}$ so that $\left\{\log \left|h_{j}\right|\right\}_{j=1}^{n}$ is a 
basis for $N^{\infty}$. Put $u_{j}=\log \left|h_{j}\right|(1 \leqslant j \leqslant n)$ and $\mathscr{E}_{0}=\left\{\exp \left(\sum_{j=1}^{n} s_{j} u_{j}\right) ; 0 \leqslant s_{j} \leqslant 1\right.$ $(1 \leqslant j \leqslant n)\}$. Then $\mathscr{E}_{0} \subseteq \mathscr{E}$. If $v$ is in $\mathscr{E}$ then there exists an $h$ in $\left(H^{\infty}\right)^{-1}$ and a $v_{0}$ in $\mathscr{E}_{0}$ such that $v=|h| v_{0}$ a.e. By Proposition 5, the supremum for all $v$ in $\mathscr{E}$ is equal to the supremum for all $v$ in $\mathscr{E}_{0}$. It follows that there exists a sequence $\left\{v_{l}\right\}$ in $\mathscr{E}_{0}$ such that $\lim _{l \rightarrow \infty} c\left(v_{l}, W\right)$ is equal to the supremum. Then $v_{l}=\exp \left(\sum_{j=1}^{n} s_{j l} u_{j}\right)$ and $0 \leqslant s_{j l} u_{j} \leqslant 1(1 \leqslant j \leqslant n)$. It follows that $s_{j l}$ converges to $s_{j}$ for each $j$ taking a subsequence if necessary, and $0 \leqslant s_{j} \leqslant 1(1 \leqslant j \leqslant n)$. Hence the supremum is attained with $v=\exp \left(\sum_{j=1}^{n} s_{j} u_{j}\right)$ in $\mathscr{E}$. This completes the proof.

5. Norms of Hankel operators. Let $Q$ be the orthogonal projection from $L^{2}$ to $\left(K^{2}\right)^{\perp}=\bar{H}_{0}^{2}$. For $\phi$ in $L^{\infty}$ and $f$ in $H^{2}$, the classical Hankel operators $H_{\phi}$ are defined by the formula

$$
H_{\phi}(f)=Q(\phi f) \text {. }
$$

The first author $[12,13]$ has given the generalizations of the classical Hankel operators. Let $v$ be a function in $L$. Let $Q_{v}$ be the orthogonal projection from $L^{2}$ to $\left(v K^{2}\right)^{\perp}=v^{-1} \bar{H}_{0}^{2}$. For $\phi$ in $L^{\infty}$ and $f$ in $v H^{2}$, the generalized Hankel operators $H_{\phi}^{v}$ are defined by the formula

$$
H_{\phi}^{v}(f)=Q_{v}(\phi f) .
$$

If $A$ is a disc algebra, then $\left\|H_{\phi}\right\|=\left\|\phi+H^{\infty}\right\|$ (see [12]). This is a theorem of Nehari. The first author [12] proved the following theorem. We shall prove it by Corollary 2.1.

THEOREM 10. Let $\phi$ be a function in $L^{\infty}$. Then

$$
\sup _{i, I .}\left\|H_{\phi}^{v}\right\|=\left\|\phi+K^{\infty}\right\| .
$$

If $m$ is a unique logmodular measure for $\tau$, then the supremum is attained with some $v$ in $\mathscr{E}$.

Proof. Put $c=\sup _{n \in L}\left\|H_{\phi}^{v}\right\|$. It follows that $c \leqslant\left\|\phi+K^{\infty}\right\|$, since $\left\|H_{\phi}^{v}(f)\right\|_{2}=$ $\left\|Q_{n}((\phi+g) f)\right\|_{2} \leqslant\|(\phi+g) f\|_{2} \leqslant\|\phi+g\|_{\infty}\|f\|_{2}$ for all $g$ in $K^{\infty}$. We shall show that $c \geqslant\left\|\phi+K^{\infty}\right\|$. By the definition of $H_{\phi}^{v}$, it follows that

$$
\left(\begin{array}{cc}
c v^{2} & \phi \\
\bar{\phi} & c v^{-2}
\end{array}\right)
$$

is positive on $A \times \overline{A_{0}}$ for all $v$ in $\mathscr{L}$. It follows from Corollary 2.1 that there exists a function $k$ in $K^{1}$ such that $|\phi+k| \leqslant c$ a.e. Hence $k$ is in $K^{1} \cap L^{\infty}=K^{\infty}$. Therefore, $\left\|\phi+K^{\infty}\right\| \leqslant c$. This completes the proof.

If $N_{\tau}$ is finite dimensional, then the supremum for "all $v$ in $\mathscr{L}$ " in Theorem 10 is equal to the supremum for "all $v$ in $\mathscr{E}$." The first author [13] proved the following theorem. We shall prove it by Theorem 7 .

THEOREM 11. Suppose $A$ is a uniform algebra such that $\gamma_{0}$ is finite. Let $\phi$ be a function in $L^{\infty}$. Then

$$
\left\|H_{\phi}\right\| \leqslant\left\|\phi+K^{\infty}\right\| \leqslant \gamma_{0}\left\|H_{\phi}\right\| .
$$


Proof. It follows from Theorem 10 that $\left\|H_{\phi}\right\| \leqslant\left\|\phi+K^{\infty}\right\|$. We prove next that $\left\|\phi+K^{\infty}\right\| \leqslant \gamma_{0}\left\|H_{\phi}\right\|$. By the definition,

$$
\left(\begin{array}{cc}
\left\|H_{\phi}\right\| & \phi \\
\bar{\phi} & \left\|H_{\phi}\right\|
\end{array}\right)
$$

is positive on $A \times \overline{A_{0}}$. It follows from Theorem 7 that there exists a $k$ in $K^{1}$ such that $|\phi+k| \leqslant \gamma_{0}\left\|H_{\phi}\right\|$ a.e. Hence $k$ is in $K^{1} \cap L^{\infty}=K^{\infty}$. Therefore, $\left\|\phi+K^{\infty}\right\| \leqslant$ $\gamma_{0}\left\|H_{\phi}\right\|$. This completes the proof.

6. The Koosis type theorem. The following theorem is a generalization of the Koosis theorem. We shall prove it by the lifting theorem.

THEOREM 12. Suppose $m$ is a representing measure for $\tau$. Let $W$ be a nonnegative integrable function.

(1) The following two conditions are equivalent.

(i) There exists a nonnegative function $U$ with $m(W U>0)>0$ such that

$$
\int_{X}|v f|^{2} U d m \leqslant \int_{X}\left|v f+v^{-1} \bar{g}\right|^{2} W d m
$$

for all $f$ in $A$, all $g$ in $A_{0}$ and all $v$ in $\mathscr{L}$.

(ii) There exists a nonzero function $k$ in $K^{1}$ such that

$$
|W-2 k| \leqslant W \text { a.e. }
$$

When the set $N_{\tau}$ is finite dimensional and $m$ is a core point of $N_{\tau}$, it is sufficient that $v$ ranges only over $\mathscr{E}$ instead of $\mathscr{L}$. Moreover, $K^{1}=H^{1}+N_{c}^{\infty}$.

(2) If there exist no nonzero functions in $K^{1}$ which vanish on a set of positive measure, then it is possible to take $U>0$ a.e. in (1).

Proof. We shall prove (1). We shall show that (i) implies (ii). It can be shown that

$$
\left(\begin{array}{cc}
v^{2}(W-U) & W \\
W & v^{-2} W
\end{array}\right)
$$

is positive on $A \times \overline{A_{0}}$ for all $v$ in $\mathscr{L}$. By Corollary 2.1, there exists a $k$ in $K^{1}$ such that $|W-k|^{2} \leqslant(W-U) W \leqslant W^{2}$ a.e. Hence, $m(k \neq 0) \geqslant m(W U>0)>0$. This implies (ii). We shall show that (ii) implies (i). Since $|W-2 k| \leqslant W$ a.e., it follows that $|k|^{2} \leqslant W \operatorname{Re} k$ a.e. Hence, $E=\{x \in X ; k \neq 0\}$ is a subset of $F=\{x \in X$; $\operatorname{Re} k>0\}$, and $m(E)>0$. Put $W_{0}=|k|^{2} /(\operatorname{Re} k)$ on $F$ and $W_{0}=0$ off $F$. Then, $\left|W_{0}-k\right|^{2}=W_{0}\left(W_{0}-\operatorname{Re} k\right)$ a.e. and $W_{0} \leqslant W$ a.e. Since $k$ annihilates $A_{0}$, for all $f$ in $A$ and all $g$ in $A_{0}$,

$$
\begin{aligned}
-2 \operatorname{Re} \int_{X} f g W_{0} d m & =-2 \operatorname{Re} \int_{X} f g\left(W_{0}-k\right) d m \\
& \leqslant 2 \int_{X}|f g| W_{0}^{1 / 2}\left(W_{0}-\operatorname{Re} k\right)^{1 / 2} d m \\
& \leqslant \int_{X}|f|^{2} v^{2}\left(W_{0}-\operatorname{Re} k\right) d m+\int_{X}|g|^{2} v^{-2} W_{0} d m .
\end{aligned}
$$


Put $U=\operatorname{Re} k$; then $U$ is a nonnegative function, and

$$
m(W U>0)=m(W \operatorname{Re} k>0)>m(E)>0 .
$$

Furthermore,

$$
\begin{aligned}
\int_{X}|v f|^{2} U d m & =\int_{X}|v f|^{2}(\operatorname{Re} k) d m \\
& \leqslant \int_{X}\left|v f+v^{-1} \bar{g}\right|^{2} W_{0} d m \leqslant \int_{X}\left|v f+v^{-1} \bar{g}\right|^{2} W d m .
\end{aligned}
$$

This implies (i). The latter half of (1) follows from Theorem 8. Then $K^{1}=H^{1}+N_{c}^{\infty}$ (cf. [6, Chapter IV, Theorem 6.2]).

Next, we shall prove (2). As the proof of (1), we can take $U=\operatorname{Re} k$ for some $k$ in $K^{1}$ such that $\operatorname{Re} k>0$ a.e. by the special property of $K^{1}$ in this case. This completes the proof.

Proposition 13. Suppose $A$ is a uniform algebra such that $\gamma_{0}$ is finite. Let $W$ be a nonnegative integrable function. If there exists a $U$ with $0 \leqslant U \leqslant W$ a.e. such that the function $s=W U-\left(1-\gamma_{0}^{-2}\right) W^{2}$ is nonnegative and nonzero, and

$$
\int_{X}|f|^{2} U d m \leqslant \int_{X}|f+\bar{g}|^{2} W d m
$$

for all $f$ in $A$ and all $g$ in $A_{0}$, then there exists a nonzero function $k$ in $K^{1}$ such that $|W-k| \leqslant W$ a.e.

Proof. It can be shown that $\left(\begin{array}{cc}W-U & W \\ W\end{array}\right)$ is positive on $A \times \overline{A_{0}}$. By Theorem 7 and the hypothesis, there exists a $k$ in $K^{1}$ such that $|W-k|^{2} \leqslant \gamma_{0}^{2}(W-U) W \leqslant W^{2}-$ $\gamma_{0}^{2} s \leqslant W^{2}$ a.e. Hence, $k$ is a nonzero function. This completes the proof.

KoOSIs' THEOREM [11]. Suppose $A$ is a disc algebra. Let $m$ be a normalized Lebesgue measure on the unit circle and let $W$ be a nonnegative integrable function. The following conditions are then equivalent.

(i) There exists a nonzero nonnegative function $U$ such that

$$
\int_{X}|f|^{2} U d m \leqslant \int_{X}|f+\bar{g}|^{2} W d m
$$

for all $f$ in $A$ and all $g$ in $A_{0}$.

(ii) There exists a nonzero function $k$ in $H^{1}$ such that $|W-2 k| \leqslant W$ a.e.

(iii) $W$ is invertible in $L^{1}$.

If the above conditions hold, there exists $U$ such that $\log U$ is integrable.

Proof. Since $A$ is a disc algebra, $\left|\left(H^{\infty}\right)^{-1}\right|=\mathscr{L}$. Hence, $\gamma_{0}=1$. Since the set $\left\{|f|^{2} ; f\right.$ is in $\left.A\right\}$ is sup-norm dense in $C(X)$, the function $U$ in (i) satisfies $U \leqslant W$ a.e. Hence, $m(U>0)=m(W U>0)$. It follows from Proposition 13 that (i) implies (ii). It follows from Theorem 12 that (ii) implies (i). Moreover, we can take $U$ such that $\log U$ is integrable, since in this case $\log (\operatorname{Re} k)$ is integrable unless $k=0$ a.e. We shall show next that condition (ii) implies (iii). It follows from (ii) that $W^{-1} \leqslant$ $\operatorname{Re}\left(k^{-1}\right)$ a.e., which implies $\operatorname{Re} k \geqslant 0$ a.e. Hence, $k$ is an outer function (cf. [7, 
Chapter II, Corollary 4.8]). It follows from Fatou's lemma that

$$
\int_{T} W^{-1} d m \leqslant \liminf _{r \rightarrow 1} \int_{0}^{2 \pi} \operatorname{Re}\left(k^{-1}\left(r e^{i \theta}\right)\right) \frac{d \theta}{2 \pi} \leqslant \operatorname{Re}\left(k^{-1}(0)\right) .
$$

Hence $W^{-1}$ is integrable which implies (iii). We shall show lastly that (iii) implies (ii). Suppose $W^{-1}$ is integrable. Put $k^{-1}=W^{-1}+i\left(W^{-1}\right)^{\sim}$, where $\left(W^{-1}\right)^{\sim}$ denotes the harmonic conjugate function of $W^{-1}$. By the calculation, $|W-2 k|=W$ a.e., which implies (ii). This completes the proof.

If a uniform algebra $A$ satisfies the condition that $A+\overline{A_{0}}$ is weak-* dense in $L^{\infty}(m)$, then the equivalence of the conditions in the above theorem remains true, since it is true also in this case that $\left|\left(H^{\infty}\right)^{-1}\right|=\mathscr{L}$.

7. The Helson-Szegö type theorem. By calculation it can be shown that if the weight $W$ satisfies the inequality

$$
\int_{X}|v f|^{2} W d m \leqslant C \int_{X}\left|v f+v^{-1} \bar{g}\right|^{2} W d m
$$

for all $f$ in $A$, all $g$ in $A_{0}$, and all $v$ in $\mathscr{L}$, then the matrix

$$
\left(\begin{array}{cc}
\left(1-C^{-1}\right) v^{2} W & W \\
W & v^{-2} W
\end{array}\right)
$$

is positive on $A \times \overline{A_{0}}$. The converse is also true. It follows from Corollary 2.1 that this condition of $W$ is equivalent to the existence of a $k$ in $K^{1}$ such that

$$
|W-2 k|^{2} \leqslant\left(1-C^{-1}\right) W^{2} \text { a.e. }
$$

For weights $W$ such that $W=|h|^{2}$ for some outer function $h$ in $H^{2}$, the following theorems are obtained by the first author (see $[12,13])$.

THEOREM 14. Suppose $m$ is a representing measure for $\tau$, and $W$ is a nonnegative integrable function. The following conditions are then equivalent.

(i) There exists a constant $C$, which is independent of $v$, such that

$$
\int_{X}|v f|^{2} W d m \leqslant C \int_{X}\left|v f+v^{-1} \bar{g}\right|^{2} W d m
$$

for all $f$ in $A$, all $g$ in $A_{0}$ and all $v$ in $\mathscr{L}$.

(ii) There exists a constant $r$ in $[0,1)$ and a function $k$ in $K^{1}$ such that $|W-2 k| \leqslant r W$ a.e. When the set $N_{\tau}$ is finite dimensional, and $m$ is a core point of $N_{\tau}$, it is sufficient that $v$ ranges only over $\mathscr{E}$ instead of $\mathscr{L}$. Moreover, $K^{1}=H^{1}+N_{c}^{\infty}$.

Proof. We shall show that (i) implies (ii). As we showed at the beginning of this section, it follows from (i) that there exists a $k$ in $K^{1}$ such that

$$
|W-2 k|^{2} \leqslant\left(1-C^{-1}\right) W^{2} \text { a.e. }
$$

Therefore we get (ii). The proof that (ii) implies (i) is as follows. Since $|W-2 k| \leqslant r W$ a.e., it follows that $\left(\left(1-r^{2}\right) / 4\right) W^{2} \leqslant|k|^{2} \leqslant W \operatorname{Re} k$ a.e. Hence,

$$
W \leqslant\left(4 /\left(1-r^{2}\right)\right) \operatorname{Re} k \text { a.e. }
$$


On the other hand, as we showed in the proof of Theorem 12, it follows that, for all $f$ in $A$ and all $g$ in $A_{0}$,

$$
\int_{X}|v f|^{2}(\operatorname{Re} k) d m \leqslant \int_{X}\left|v f+v^{-1} \bar{g}\right|^{2} W d m .
$$

Put $C=4 /\left(1-r^{2}\right)$, then (i) follows. This completes the proof.

When $A$ is a disc algebra, the original Helson-Szegö theorem [8] follows from Theorem 14. The proof of the equivalence of the boundedness of the Riesz projection and condition (ii) in Theorem 14 is almost the same as the proof of Koosis' theorem. We should mention that the condition $\left\|\exp \left(-i(\log W)^{\sim}\right)+H^{\infty}\right\|<$ 1 is also equivalent in this case.

THEOREM 15. Suppose $m$ is a unique logmodular measure for $\tau$, and $W$ is a nonnegative integrable function. If for each $v$ in $\mathscr{L}$, there exists a constant $C_{v}$ such that

$$
\int_{X}|v f|^{2} W d m \leqslant C_{v} \int_{X}\left|v f+v^{-1} \bar{g}\right|^{2} W d m
$$

for all $f$ in $A$, all $g$ in $A_{0}$, then $\sup _{v \in \mathscr{L}} C_{v}<\infty$.

Proof. Put $W_{11}=\left(1-C_{v}^{-1}\right) W$ and $W_{12}=W_{21}=W_{22}=W$. It follows from Theorem 9 that there exists a function $v_{0}$ in $\mathscr{L}$ such that (ii) holds with $C=C_{v_{0}}$. This completes the proof.

Let $P$ be the $L^{2}(m)$-orthogonal projection from $A+\overline{A_{0}}$ to $A$. Put

$$
\begin{gathered}
\|P\|_{W}=\inf \left\{c \geqslant\left. 0\left|\int_{X}\right| f\right|^{2} W d m \leqslant c^{2} \int_{X}|f+\bar{g}|^{2} W d m\right. \\
\left.\quad \text { for all } f \text { in } A \text { and all } g \text { in } A_{0}\right\}, \\
\rho(W)=\inf \left\{c \geqslant 0 ;|W-k| \leqslant c W \text { a.e. for some } k \text { in } K^{1}\right\} .
\end{gathered}
$$

If $A$ is a disc algebra, then $\rho(W)=\left\|\exp \left(-i(\log W)^{-}\right)+H^{\infty}\right\|$. This is also equal to the cosine of the angle between $A$ and $\overline{A_{0}}$ in the weighted Hilbert space $L^{2}(W)$. Then $\rho(W)<1$ if and only if $\|P\|_{W}<\infty$.

THEOREM 16. Let $W$ be a nonnegative function in $L^{1}$. Then

$$
\left\{1-\left(\frac{1}{\|P\|_{W}}\right)^{2}\right\}^{1 / 2} \leqslant \rho(W) \leqslant \gamma_{0}\left\{1-\left(\frac{1}{\|P\|_{W}}\right)^{2}\right\}^{1 / 2} .
$$

Proof. By the calculation at the beginning of this section and by Theorem 7 , this theorem follows.

8. Examples and appendix. All results in this paper were known in a disc algebra. But they also apply to many other concrete examples.

(1) We assume that $Y$ is a compact subset of the complex plane whose complement has a finite number of components. The algebra $R(Y)$ consists of all functions in $C(Y)$ which can be approximated uniformly on $Y$ by rational functions with poles off $Y$. Let $A$ be the restriction of $R(Y)$ to its Shilov boundary $X$. Then the 
uniform closure of $\operatorname{Re} A$ has finite codimension in $C_{R}(X)$, and the linear span of $\log \left|A^{-1}\right|$ is dense in $C_{R}(X)$ (cf. [6, Chapter IV, Theorem 8.3]). Hence, every $\tau$ in $M_{A}$ has a finite-dimensional set $N_{\tau}$ of representing measures, and every $\tau$ in $M_{A}$ has a unique logmodular measure $m$ in $N_{\tau}$. Then $m$ is a core point of $N_{\tau}$ (cf. [6, Chapter IV, Corollary 7.5]) and $\gamma_{0}$ is finite. When $Y$ is an annulus $\{r \leqslant|z| \leqslant 1\}$, then $\gamma_{0}=r^{-1 / 2}$ (cf. [13]).

(2) Let $A$ be a subalgebra of the disc algebra which contains the constants and which has finite codimension in the disc algebra. Two examples of such subalgebras are $\left\{f ; f^{\prime}(0)=0\right\}$ and $\{f ; f(0)=f(1 / 2)\}$. If $\tau(f)=f(0)$ for $f$ in $A$ and $m$ is the normalized Lebesgue measure on the unit circle $\mathbf{T}$, then $N_{\tau}$ is finite dimensional, $m$ is a core point of $N_{\tau}, N^{\infty} \subseteq C(\mathbf{T})$ and $\gamma_{0}$ is infinite (cf. [13]).

(3) The unit polydisc $\mathbf{U}^{n}$ is defined to be the set of all $z$ in $\mathbf{C}^{n}$ whose coordinates satisfy $\left|z_{i}\right|<1$ for $i=1, \ldots, n$. For simplicity, we assume $n=2$. Part of the boundary of $\mathbf{U}^{2}$ is the torus $\mathbf{T}^{2}$, the set of all $(z, w)$ in $\mathbf{C}^{2}$ with $|z|=|w|=1$. Let $A\left(\mathrm{U}^{2}\right)$ be the class of all continuous complex-valued functions on the closure of $\mathrm{U}^{2}$ which is holomorphic in $\mathbf{U}^{2}$. Let the polydisc algebra $A$ be the restriction of $A\left(\mathbf{U}^{2}\right)$ to $\mathbf{T}^{2}$. If $\tau(f)=f(0,0)$ for $f$ in $A$ and $m_{2}$ is the normalized Lebesgue measure on $\mathbf{T}^{2}$, then $\tau$ is in $M_{A}$ and $m_{2}$ is a representing measure for $\tau$. The Fourier coefficients $\hat{f}(n, m)$ of an integrable function $f$ are defined for all integers $n$ and $m$ by

$$
\hat{f}(n, m)=\int_{\mathbf{T}^{2}} f(z, w) \bar{z}^{n} \bar{w}^{n} d m_{2}(z, w) .
$$

Let $\mathbf{Z}_{+}^{2}$ denote the set of all pairs $(n, m)$ of nonnegative integers $n$ and $m$. Let $C\left(\mathbf{T}^{2}\right)$ be the algebra of continuous complex-valued functions on $\mathbf{T}^{2}$. Then $A=\{f \in$ $C\left(\mathbf{T}^{2}\right) ; \hat{f}(n, m)=0$ for $(n, m)$ off $\left.\mathbf{Z}_{+}^{2}\right\}$. For $1 \leqslant p \leqslant \infty, H^{p}=\left\{f \in L^{p} ; \hat{f}(n, m)\right.$ $=0$ for $(n, m)$ off $\left.\mathbf{Z}_{+}^{2}\right\}$ and $K^{p}=\left\{f \in L^{p} ; \hat{f}(n, m)=0\right.$ for $(-n,-m)$ in $\left.\mathbf{Z}_{+}^{2}\right\}$.

Throughout this paper we have studied the measure matrices which are positive on $A \times \overline{A_{0}}$. There is another kind of lifting theorem. Let $K=K^{\infty} \cap C(X)$ and $K_{0}=K_{0}^{\infty} \cap C(X)$. All results in this paper are valid for $A \times \bar{K}_{0}$ instead of $A \times \overline{A_{0}}$. The following Theorem 2 ' corresponds to Theorem 2. The definition of the relation $\mu \sim \nu$ for measure matrices $\mu$ and $\nu$ in this case is different from the definition which was given in the Introduction. If two measure matrices $\mu=\left(\mu_{i j}\right)$ and $v=\left(\nu_{i j}\right)$ satisfy $\mu_{11}=\nu_{11}, \mu_{22}$ and $\mu_{12}-\nu_{12}$ annihilates $K_{0}$, then we write $\mu \sim \nu$. If $\mu \sim \nu$, then $\mu=\nu$ on $A \times \bar{K}_{0}$.

TheOREM $2^{\prime}$. Let $A$ be a uniform algebra on $X$, and let $\tau$ be in $M_{A}$. Suppose a measure matrix $\mu=\left(\mu_{i j}\right)$ satisfies condition (\#). Let

$$
d \mu_{i j}=W_{i j} d m+d \mu_{i j}^{s} \quad(i, j=1,2)
$$

be its Lebesgue decomposition with respect to $N_{\tau}$. The following conditions are then equivalent.

(i) All $\lambda$ in $[\mu]$ are positive on $A \times \bar{K}_{0}$.

(ii) There exists a positive measure matrix $v=\left(\nu_{i j}\right)$ on $C(X) \times C(X)$ such that $\mu \sim \nu$ and $\left(\mu_{i j}^{s}\right)=\left(\nu_{i j}^{s}\right)$.

As an application of Theorem $2^{\prime}$, for example, the following Theorem 14' holds. Let $K_{0}^{\perp} \cap L^{1}=\left\{h \in L^{1} ; \int_{X} h k d m=0\right.$ for all $k$ in $\left.K_{0}\right\}$. 
THEOREM 14'. Suppose $m$ is a representing measure for $\tau$ and $W$ is a nonnegative integrable function. The following conditions are then equivalent.

(i) There exists a constant $C$, which is independent of $v$, such that

$$
\int_{X}|v f|^{2} W d m \leqslant C \int_{X}\left|v f+v^{-1} \bar{g}\right|^{2} W d m
$$

for all $f$ in $A$, all $g$ in $K_{0}$ and all $v$ in $\mathscr{L}$.

(ii) There exists a constant $r$ in $[0,1)$ and a function $k$ in $K_{0}^{\perp} \cap L^{1}$ such that $|W-2 k| \leqslant r W$ a.e. If $K_{0}$ is weak-* dense in $K_{0}^{\infty}$, then $K_{0}^{\perp} \cap L^{1}$ coincides with $H^{1}$. Examples (1), (2) and (3) satisfy this condition.

We should remark on condition (\#) in the Introduction. If $N_{\tau}$ is finite dimensional, and $\tau$ has a unique logmodular measure $m$, then $\mathscr{L}$ is contained in $\left|H^{\infty}\right|=\left\{|h| ; h\right.$ is in $\left.H^{\infty}\right\}$. Hence, $\left|H^{\infty}\right|$ is uniformly dense in the set of essentially bounded nonnegative functions (cf. [6, Chapter IV, Exercises 11 and 12]). The matrix $\mathbf{W}=\left(W_{i j}\right)$, which is positive on $A \times \overline{A_{0}}$, satisfies the conditions $W_{11} \geqslant 0$ a.e. and $W_{22} \geqslant 0$ a.e. This is applicable to Examples (1) and (2). If $A$ is a polydisc algebra as in Example (3), then $|A|$ is uniformly dense in the set of continuous nonnegative functions. Hence, if the matrix $\mu=\left(\mu_{i j}\right)$ is positive on $A \times \overline{A_{0}}$, then it satisfies the conditions $\mu_{11} \geqslant 0$ and $\mu_{22} \geqslant 0$.

\section{REFERENCES}

1. R. Arocena and M. Cotlar, A generalized Helglotz-Bochner theorem and $L^{2}$-weighted inequalities with finite measures, Conference on Harmonic Analysis in Honor of Antoni Zygmund (Chicago, Ill., 1981), Wadsworth Math. Ser., Wadsworth, Belmont, Calif., 1983, pp. 258-269.

2. R. Arocena, M. Cotlar and C. Sadosky, Weighted inequalities in $L^{2}$ and lifting properties, Adv. in Math. Suppl. Studies 7A, Academic Press, New York, 1981, pp. 95-128.

3. K. Barbey and H. König, Abstract analytic function theory and Hardy algebras, Lecture Notes in Math., vol. 593, Springer-Verlag, Berlin, 1977.

4. M. Cotlar and C. Sadosky, On the Helson-Szegö theorem and a related class of modified Toeplitz kernels, Harmonic Analysis in Euclidean Spaces (Williamstown, Mass., 1978), Proc. Sympos. Pure Math., vol. 35, Amer. Math. Soc., Providence, R.I., 1979, pp. 383-407.

5. L. Lifting properties, Nehari theorem, and Paley lacunary inequality, Rev. Mat. Iberoamericana 2 (1986), 55-72.

6. T. (jamelin, Uniform algebras, 2nd ed., Chelsea, New York, 1984.

7. J. B. Garnett, Bounded analytic functions, Academic Press, New York, 1981.

8. H. Helson and G. Szegö, A problem in prediction theory, Ann. Mat. Pura Appl. 51 (1960), 107-138.

9. I. I. Hirschman, Jr. and R. Rochberg, Conjugate function theory in weak* Dirichlet algebras, J. Funct. Anal. 16 (1974), 359-371.

10. P. Koosis, Weighted quadratic means of Hilhert transforms, Duke Math. J. 38 (1971), 609-634.

11. , Movennes quadratiques pondérées de fonctions périodiques et de leurs conjuguées har:noniques,

C. R. Acad. Sci. Paris 291 (1980), 255-257.

12. T. Nakazi, Norms of Hankel operators and uniform algebras, Trans. Amer. Math. Soc. 299 (1987), $573-580$.

13. Norms of Hankel operators and uniform algebras. II, Tôhoku Math. J. (to appear).

14. Y. Ohno, Remarks on Helson-Szegö problems, Tôhoku Math. J. 18 (1966), 54-59.

15. T. Yamamoto, On the generalization of the theorem of Helson and Szegö, Hokkaido Math. J. 14 (1985), 1-11.

Department of Mathematics, Faculty of Science (General. Education), Hokkaido UniverSITY, SAPPORO 060, JAPAN

Department of Mathematics, faculty of Generai. Education, Hokkai - Gakuen University, SAPPORO 062, JAPAN 